\title{
Protée
}

\section{Texture, couleur, lumière et autres arrangements de la perception}

\section{Anne Beyaert}

Volume 31, numéro 3, hiver 2003

Lumières

URI : https://id.erudit.org/iderudit/008439ar

DOI : https://doi.org/10.7202/008439ar

Aller au sommaire du numéro

Éditeur(s)

Département des arts et lettres - Université du Québec à Chicoutimi

ISSN

0300-3523 (imprimé)

1708-2307 (numérique)

Découvrir la revue

Citer cet article

Beyaert, A. (2003). Texture, couleur, lumière et autres arrangements de la perception. Protée, 31(3), 81-90. https://doi.org/10.7202/008439ar

\section{Résumé de l'article}

L'article entend faire l'éloge d'une dimension généralement considérée comme ancillaire dans les arts visuels, la texture. Il distingue tout d'abord la texture représentée (la chair du monde) de la texture ostensive (la chair de la peinture) et, sur ces bases, examine les relations qu'entretiennent les différentes dimensions du visible et les liens du visible au tangible. Enfin, il montre une accentuation de la texture dans l'art du $\mathrm{xx}^{\mathrm{e}}$ siècle, chez les surréalistes et dans l'installation, notamment. 


\title{
TEXTU RE, CO U LEU R, LU M IÈRE ET AUTRES ARRANGEMENTS DE LA PERCEPTION
}

AN NE BEYAERT

\begin{abstract}
Je recule devant la difficulté en élevant un palais d'abstractions. Je bronche devant l'obstacle, comme tant ont peur de l'autre et de sa peau. Comme tant ont peur de leurs sens et réduisent à rien, à la table rase de l'immangeable, la somptueuse queue de paon virtuelle et pliée du goûter.
\end{abstract}

Michel Serres ${ }^{1}$

Dans son effort pour différencier, catégoriser, hiérarchiser, la sémiotique tend à présenter la couleur, la texture, la lumière et la forme comme des dimensions autonomes de l'objet. Sitôt soumise à l'examen, chacune de ces dimensions discrètes s'avère pourtant inséparable des autres, qu'elle instruit et modifie: la lumière définit la couleur qui se rapporte à une texture, détermine elle-même la dimension et la luminosité de la plage chromatique. Toutes les dimensions se tiennent dans la perception, même si la visée heuristique contraint la sémiotique à marquer les contrastes, à «exagérer», comme l'indiquait déjà E. Cassirer.

On ne parvient en réalité aux data de la "simple» sensation - comme clair ou sombre, chaud ou froid, rugueux ou lisse -, soutenait en effet cet auteur, qu'en laissant de côté une couche fondamentale et primitive de la perception, en la déblayant pour ainsi dire dans une intention théorique précise. ${ }^{2}$

Cet article a pour objet d'étude la «couche fondamentale de la perception" hâtivement déblayée et s'attache plus précisément à une dimension ancillaire de l'objet de sens, la texture. Il souhaite marquer la place de cette dimension au cœur du visible et distinguer ses deux acceptions: la texture représentée et la texture présentée ou ostensive. Un tel éloge amène enfin à reconsidérer l'importance de la texture dans l'art du XXe siècle.

J. Ninio définit la texture comme «le grain des choses, la qualité la plus tangible des objets, bien avant leur couleur ou leur forme " ${ }^{3}$. C'est la qualité visuelle qui permet d'identifier un matériau: métal, étoffe, bois, etc. À la différence des couleurs qui se laissent précisément classer et décrire (saturation, 
tonalité, teinte), les qualités texturales passent au crible d'un vocabulaire disparate et mal organisé: rêche, grenu, ramé, nacré, frisé, etc. Le biologiste décrit la perception des textures sur fond de correspondance entre le visuel et le tactile:

[...] l'œil, dit-il, ne peut séparer les centaines de milliers de cheveux

d'une tresse, et pourtant la sensation de les voir un à un est nette.

[...] [Le] cerveau invente! Il crée littéralement, à partir de jeux

d'ombre et de lumière sur la coiffe, une texture de cheveux

procurant une sensation de surface ondulante très finement divisée.

Sans jamais dénommer la correspondance intersensorielle, Ninio développe donc une conception synesthésique de la texture fonctionnant par transposition visuelle. En termes similaires, F. SaintMartin a décrit la texture comme une fiction qui évoque le tactile en exploitant toutes les dimensions du visible. Elle explique:

[...] l'art figuratif a [...] largement exploité les possibilités

fictionnelles de la représentation de l'expérience tactile par

l'élaboration de textures picturales dissociées de leur référence.

Souvent, une matière relativement lisse donnera, par le jeu des

tonalités, des éclairages, des glissements entre formes et couleurs,

l'illusion de textures rugueuses, chaotiques, poilues, etc. 4

Une telle conception trouve encore écho chez le Groupe $\mu$ pour qui le terme texture, dérivé d'un mot latin signifiant "tissu», se réfère «au grain de la surface d'un objet ou à l'espèce de sensation tactile qu'il produit visuellement " ${ }^{5}$. Pour ces auteurs, la texture trouve donc une origine synesthésique, donnée pour telle, et suppose une équivalence entre les deux modalités perceptives ${ }^{6}$.

Si la texture se conçoit comme une synthèse des sens, une synesthésie, sa définition ne se départit pas, chez les auteurs de Liège comme chez Saint-Martin, d'une acception analytique, le terme désignant alors une «microtopographie, constituée par la répétition d'éléments" 7 . C'est une granularité (Groupe $\mu$ ) particulière ou une granulosité (Saint-Martin). Suivant cette voie, Saint-Martin fait ainsi de la texture une propriété attachée à la surface comme à la structure interne du pigment:
Par le terme de texture, on entend une propriété de la masse colorée qui joue aussi bien dans ses profondeurs que sur la pellicule par des inclinaisons diverses et des disjonctions qui infléchissent différemment l'absorption et la réfraction des rayons lumineux par des corps opaques, modifiant ainsi leurs effets chromatiques. ${ }^{8}$

\section{LES DEUX VERSANTS DE LA TEXTURE}

Cette brève recension révèle deux acceptions de la texture. L'une qui définit l'apparence d'un objet représenté - fruits, fleurs ou ailes de mouche -, ce qui se laisserait décrire comme "la chair du monde", l'autre qui renvoie à une propriété de surface du tableau, c'est-à-dire la «chair de la peinture». D'un côté, la texture est une fiction $d u$ monde offerte à la perception; de l'autre, c'est une propriété de la surface.

Une autre façon de traiter cette dichotomie serait encore d'opposer un régime de représentation de la texture à un régime de présentation ou d'ostension; mais, en filigrane, ces conceptions subjective et objective de la texture ne cesseraient de rappeler les débats, infiniment plus amples, sur la couleur conçue, elle aussi, tantôt comme matière colorique, tantôt comme sensation chromatique 9 .

"Chair du monde" ou "chair de la peinture», on pourrait s'interroger sur le bien-fondé de telles distinctions, dans la mesure où nos efforts théoriques sont nécessairement brisés par l'expérience perceptive qui estompe les différences et les soumet nécessairement à la synthèse du corps-propre. En outre, et de la même façon que les sémioticiens tendent à confondre les deux régimes épistémologiques de la texture, les peintres n'ont cessé d'entremêler les deux acceptions, la texture représentée et la texture ostensive. À titre d'exemple, nous pourrions évoquer les portraits du peintre britannique Glenn Brown, dérivés d'œuvres célèbres, qui, en exagérant les contrastes texturaux et chromatiques, confondent la chair de la peinture et la chair de l'être représenté: le visage peint d'après l'autoportrait de Rembrandt est-il affligé de profondes cicatrices ou s'agit-il des marques d'une énonciation? 


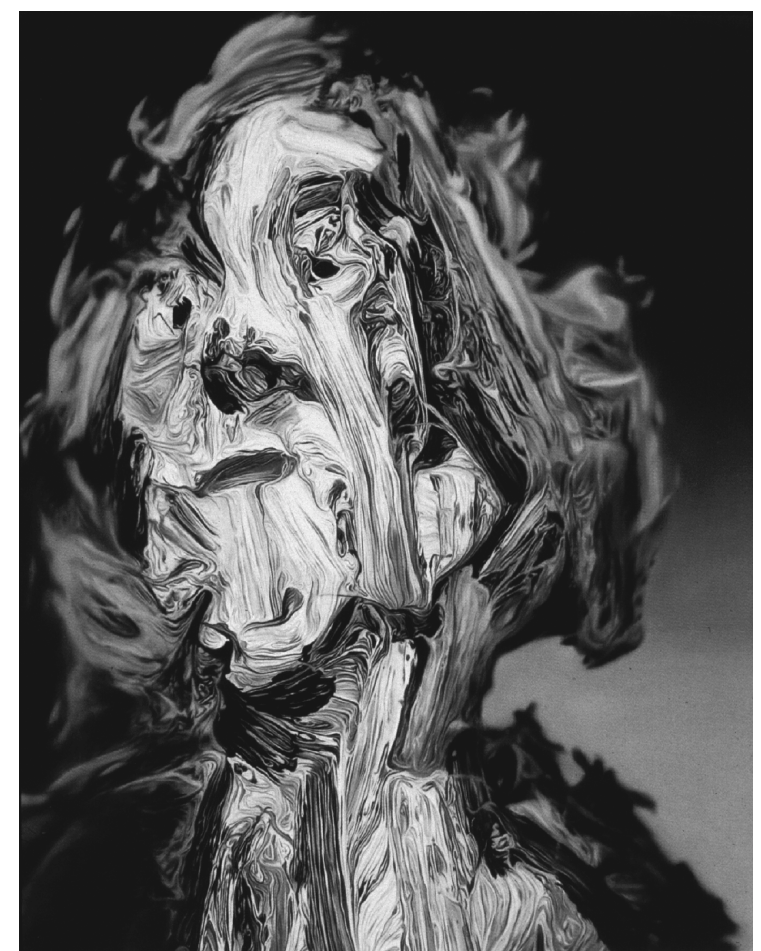

Glenn Brown, Kill the Poor, huile sur toile, 71 x $57 \mathrm{~cm}, 2000$. (C) DACS (Londres) / SODART (Montréal) 2003.

Et cet homme, plus proche désormais de Frankenstein que du portrait initial fait par Auerbach, est-il un monstre de peinture ou un pauvre hère balafré, dont le portrait s'applique à restituer les blessures? La confusion entre texture représentée et texture ostensive, que marquait déjà le Boeuf écorché de Rembrandt, assume en tout cas une visée eschatologique et permet de témoigner, par les moyens de la peinture, de la fragilité de l'être.

Est-il nécessaire de marquer une différence que la sémiotique et que l'art lui-même tendent à atténuer, sinon à entretenir? Poser la dichotomie nous permettra de préciser la dépendance de la texture vis-àvis des autres dimensions du visible, vis-à-vis des autres dimensions du sensible. Cette dichotomie s'impose comme une prémisse essentielle à notre éloge de l'art d'aujourd'hui.

\section{LA TEXTURE ET LA LUMIÈRE}

Si la texture assure le lien entre le visuel et le tactile, comme l'indique son fondement synesthésique, elle témoigne aussi d'un certain accord du visible lui- même. Elle dépend de la lumière qui permet d'établir la régularité et de différencier les textures. La relation à la lumière est entièrement déterminée par la dichotomie /représentation versus ostension/ et s'incarne dans le concept de lumière-matière, l'un des quatre états constitutifs de la configuration de la lumière selon J. Fontanille ${ }^{10}$. Dans le cas de la texture ostensive, la lumière-matière rencontre les discontinuités de surface et produit des ombres; dans le cas de la texture représentée, la lumière-matière n'est que fiction et représente les discontinuités de surface par des différences tonales. Ainsi représente-t-elle la «chair du monde» par des fictions révélatrices d'un «style», d'un certain rapport au monde. Gombrich, par exemple, a décrit un telle transformation de la fiction tonale au XVII ${ }^{\mathrm{e}}$ siècle, quand, au lieu de dessiner «chaque point d'aiguille ou tout au moins de nous en donner l'impression", Rembrandt découvrit que "c'était là peine inutile si les touches lumineuses étaient habilement disposées" ${ }^{11}$.

Copie ou fiction de la chair du monde, la texture s'exprime en tout cas par une forme modulaire constituée d'unités et par une loi de répétition de ces unités. Parmi d'autres auteurs, le Groupe $\mu$ a rendu compte des résultats de Bela Julesz relatifs aux lois statistiques de production des textures et distingue les textures selon leur luminance et leur granularité 12 .

Définir la texture comme une répétition d'unités produisant une discontinuité tonale présente quelques avantages majeurs. Tout d'abord, cette conception permet de dégager la texture de toutes les connotations d'épaisseur qui lui sont attachées: une texture peut être épaisse et satisfaire une conception «matiériste» à la façon de Tapiès, par exemple, mais elle peut tout aussi, sans sacrifier sa définition, présenter la fine granulosité d'une peinture de Rothko.

De façon plus essentielle, concevoir la texture comme une trame tonale régulière permet d'élargir son aire d'influence pour aborder, au-delà de l'inévitable référence à la peinture figurative, des corpus tels que l'image photographique ou audiovisuelle. La définition permet, par exemple, de décrire les images récentes produites par 
numérisation, comme les productions graphiques de John Maeda, qui procèdent par itération d'une unité programmatique - capuchon de marqueurs ou matériel informatique miniaturisé - pour reconstituer le visage d'une Joconde (publicité pour la marque Too), un tournesol (publicité Seibu) ou un volcan (publicité Sony). L'artiste-informaticien a d'ailleurs décrit le mode d'élaboration de ses images numériques et énoncé deux points de méthode qui rappellent le mode d'élaboration de la texture: le choix d'une unité programmatique et celui d'une loi de répétition. Maeda précise d'ailleurs:

[...] une forme programmatique correctement construite est par définition de nature variable, mais si l'idée visuelle centrale est médiocre, ses variations le seront également. ${ }^{13}$

Déterminée par deux attracteurs iconiques, l'icône de base (le minuscule marqueur infiniment répété) et l'icône finale (le portrait de la Joconde), l'élaboration de l'image numérique de Maeda se laisse ainsi concevoir comme une production texturale fondée sur la définition d'une unité et sa réplication.

\section{LA TEXTURE ET LE MODELÉ}

Définir la texture comme une trame tonale régulière permet enfin de croiser la notion de modelé dont elle se distingue par de nombreux points. Si la texture est une fiction du matériau de l'objet (verre, métal, etc.), le modelé impose la fiction du volume de l'objet. A. Lhote, qui fut sans doute son plus farouche détracteur, le considère comme «le plus banal, le plus usé des procédés d'imitation» ${ }^{14}$. Le modelé correspond à l'ombrage de Léonard, plus précisément à l'ombrage différentiel situé sur les côtés de l'objet ${ }^{15}$, et plus simplement à la pénombre, décrite comme "partie comprise entre le clair et le sombre où l'on passe dans l'autre en atténuant peu à peu la rotondité de l'objet" dans la terminologie simplifiée de Gombrich ${ }^{16}$.

$\mathrm{Si}$, sur le plan de l'expression, modelé et texture s'expriment par des trames tonales, la parenté ne résiste pas à l'examen. Les deux dimensions supposent des notations conventionnelles distinctes et des systèmes semi-symboliques qui diffèrent sur leurs deux plans. La texture suppose en effet une structure discrète et continue, tandis que le modelé utilise des valeurs graduelles, un dégradé, seul susceptible de restituer l'insertion de l'objet dans la profondeur.

$$
\frac{\text { texture }}{\text { modelé }}=\frac{\text { valeurs discrètes }}{\text { valeurs graduelles }}=\frac{\text { matériau }}{\text { volume }}
$$

La théorie permet donc de distinguer la représentation de la texture de celle du modelé. Pourtant, l'observation des œuvres montre, au contraire, comment les deux projets sémiotiques s'entremêlent et instruisent des fictions complémentaires. À propos de deux versions d'un bois gravé de Baldung Grien représentant La Chute de l'homme, Gombrich a montré, par exemple, comment des nuances tonales précisent à la fois la représentation du modelé et celle de la texture de la peau. Il explique:

[...] ces indications lumineuses, non seulement donnent plus de relief au modelé, mais elles en indiquent également ce que nous appelons la «texture», c'est-à-dire la façon dont se comporte la lumière qui rencontre une surface déterminée. Ce n'est donc que cette version en demi-teintes qui sera capable de nous donner l'impression du corps écailleux d'un serpent. 17

S'ils constituent des notations conventionnelles distinctes et parfaitement établies, modelé et texture peuvent donc, le cas échéant, croiser leurs efforts et se superposer pour conjuguer leurs effets de sens. Dans le cas du serpent décrit par Gombrich, la trame tonale représente à la fois la rotondité serpentine et la peau écailleuse, sachant qu'une information texturale ponctuelle, agissant à la façon d'une métonymie, suffit à décrire l'apparence de la peau.

\section{TEXTURE, COULEUR, DIMENSION}

Dans le cas de la texture ostensive, la dépendance à la lumière apparaît plus clairement encore et ne peut être séparée de critères chromatiques et même dimensionnels. Cette intrication des dimensions du visible a été soulignée par M. Guillot: 
Suivant qu'un pigment sera pulvérisé de façon plus ou moins fine, sa couleur changera... d'abord à mesure que le produit devient plus fin, la surface augmente. Quand les grains sont gros, on a l'impression d'un objet très coloré; quand les grains sont petits, il y a beaucoup de surfaces réfléchissantes, la poudre réfléchit beaucoup de lumière blanche et elle paraît plus claire. 18

En somme, et à suivre ce fin praticien de la peinture, la dépendance de la texture aux autres dimensions imposerait un triple système tensif, qui corrèle épaisseur texturale et dimension de la plage de façon inverse (l'épaisseur réduit l'étendue figurative et produit un effet de compacité), épaisseur et saturation de façon converse (l'épaisseur intensifie la couleur), tandis qu'épaisseur et tonalité progressent, de même, symétriquement (l'épaisseur assombrit):

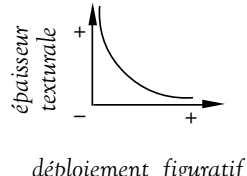

déploiement figuratif

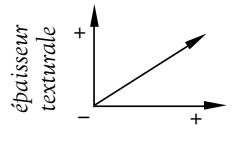

saturation

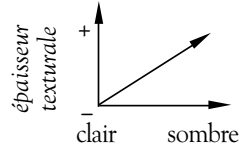

tonalité
De nombreux exemples, tirés de notre expérience du monde naturel ou de l'histoire de l'art, pourraient valider ces données perceptives: la granulosité extrêmement fine de la peinture de Rothko, par exemple, n'est sans doute pas sans incidence sur la luminosité et l'impression d'enveloppement que procurent ses vastes tableaux.

L'incidence de la texture sur la couleur et la dimension d'une plage apparaît de même dans le traitement de sa limite. Saint-Martin évoque cette inférence:

[...] lorsque le contour d'une masse est net, par suite d'un changement brusque du type de stimulation, sa couleur se présente comme dense et intense, mais quand le contour ou la frontière est indéfinie, par suite d'un changement graduel du type de stimulation, la masse chromatique devient diffuse, poudreuse et comme recouverte d'un voile de fumée. 19

Si la définition du contour peut modifier la texture d'une plage, la proposition inverse pourrait également être faite: la qualité d'une texture modifie la limite. C'est ce qu'indique Ninio, par exemple, qui observe que les lignes les plus saillantes délimitent toujours deux textures dans le monde naturel: le contour d'un meuble fera un contraste fort s'il se détache sur le papier peint d'un mur. De même, la ligne de côte qui sépare l'eau de la terre nous apparaît toujours comme soulignée d'un cerne noir lorsque nous la voyons d'un avion ou d'un bateau 20 .

\section{L'EXEMPLE DE MALEVITCH}

Un exemple suffit à restituer cette intrication de la texture et des dimensions du visible. L'œuvre de K. Malevitch, Suprématisme, Blanc sur blanc (1917), se conçoit sans réserve comme une accentuation de la dominante texturale du visible; une variation intentionnelle caractéristique du suprématisme ayant permis d'abaisser la dominante éidétique - le suprématisme a été glosé comme un «zéro des formes» - au profit d'une dominante texturale. Pourtant, si la texture commande bien l'objet de sens, le contraste des textures détermine l'éidétique. Il produit un contour séparant une figure - la plage accentuée dans l'expérience perceptive - d'un fond, qui n'est pas accentué. Ce contraste de deux textures correspond en outre à des granularités différentes en surface, c'està-dire à deux façons de réfléchir la lumière et de stabiliser la couleur.

Si la texture reste étroitement liée aux autres dimensions du visible, elle dépend en première instance de la matière. Tous les auteurs s'accordent à définir la couleur comme le résultat de la rencontre de la lumière et de la matière, un accord lumière-matière. Saint-Martin la décrit comme «le produit d'un phénomène perceptif, localisé sur les interfaces de la matière qui sont en contact avec l'air parce qu'elles sont en rapport avec la lumière» ${ }^{21}$, ce que corrobore cette élégante définition de Monnier et Saksis: «les couleurs sont un effort de la matière pour devenir une lumière "22. Si les liens de la texture et de la matière ne retiennent guère l'attention, on pourrait aisément leur donner une définition approchante: la texture serait alors un effort de la matière pour manifester la lumière. 
Plus précisément, l'opération fondamentale correspond à la rencontre de la lumière et de la matière qui, selon les propriétés des deux instances en présence - c'est-à-dire selon l'obstacle opposé à la propagation de la lumière et les propriétés de l'éclairage ${ }^{23}$-, stabilise un visible composé d'éidétique, de texture, de couleur et de lumière. Structure modulaire restituant une discontinuité tonale (texture représentée) ou lumineuse (texture ostensive), la texture produit alors, selon le cas, la fiction d'une interférence lumineuse ou l'interférence lumineuse elle-même.

\section{UN SIĖCLE DES TEXTURES}

La distinction entre la texture représentée et la texture ostensive étant faite, nous pourrions définir le XXe siècle comme un siècle des textures. Une telle assertion prendrait le contre-pied des thèses de J. $\mathrm{Ninio}^{24}$, qui considère les peintres $\mathrm{du}_{\mathrm{XV}}$, primitifs flamands ou italiens, comme des "peintres de la texture». Il célèbre l'habileté de ces artistes à représenter toutes les qualités texturales, méthodiquement rassemblées dans un même tableau, et dénie cette capacité à l'art d'aujourd'hui. Certes, la représentation de la diversité texturale du monde naturel n'est plus un critère d'appréciation des œuvres comme elle le fut alors, et l'effort des peintres ne porte plus sur la parfaite représentation des fruits, des dentelles et des ailes de mouche, mais cette réserve n'exclut pas un souci d'accentuation de la texture. À l'instar du XVe siècle, le XXe siècle se laisserait alors identifier à un siècle des textures, un glissement épistémologique déplaçant l'accent de la texture représentée (la chair du monde) à la texture ostensive (la chair de la peinture).

Plusieurs exemples manifestent un tel glissement et confirment la domination de la texture. Si elle se manifeste avec la plus grande évidence dans l'œuvre de Malevitch déjà citée, l'accentuation de la texture trouve une manifestation systématique chez Van Gogh: par exemple, lorsqu'il représente le paysage, il fait du blé une épaisseur tressée et chahutée par le vent. Elle apparaît, de façon plus évidente encore,

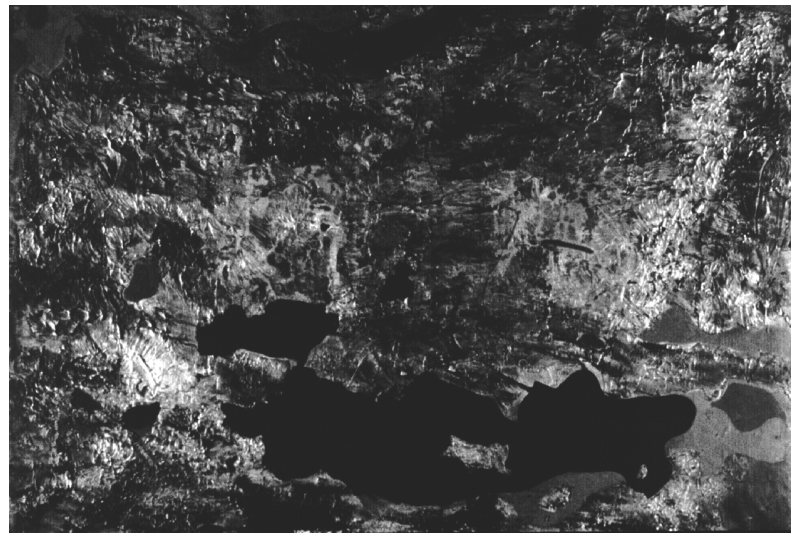

Max Ernst, L'Europe après la pluie, 101 x $149 \mathrm{~cm}, 1933$. (c) Succession Max Ernst/ADAGP (Paris) / SODRAC (Montréal) 2003.

chez les surréalistes qui, selon le cas, font prévaloir par cette entremise un rapport iconique au monde naturel (chez Magritte, la texture du bois d'une porte ou d'un mur de brique est considérablement grossie, comme par un effet de zoom) ou un rapport indiciel (les frottages de Max Ernst ${ }^{25}$ ). La texture commande alors la représentation de l'objet et, renouvelant l'organisation du visible, décrit un autre objet de sens.

Les collages surréalistes ${ }^{26}$ présentent l'intérêt de réunir les deux régimes épistémologiques de la texture. Tantôt ils reproduisent la texture, tantôt ils présentent un fragment de l'objet lui-même, une feuille de journal par exemple: la texture ostensive. Présenté plutôt que représenté, l'objet agit alors "comme un véritable double comportant la modification maxima propre au double», selon l'expression de Deleuze ${ }^{27}$.

La valorisation du textural peut s'incarner dans la représentation d'une hypoicône en attente de stabilisation, un attracteur iconique venant le plus souvent en appui. L'exemple le plus célèbre est sans doute celui de W. Turner. Délaissant l'aquarelle et la peinture à l'huile pour explorer la plasticité de l'image numérique, nous pourrions ajouter certaines photographies et images télévisuelles actuelles 28 fondées sur les possibilités allusives du crénelage.

Enfin, une autre façon d'asseoir la suprématie texturale consiste à «ensevelir» la figure. L'exemple serait alors le peintre Eugène Leroy ${ }^{29}$ qui, dans un premier temps, trace une figure avant de la recouvrir 
de couches successives et discontinues à la façon du héros du roman de Balzac, Le Chef-d'œuvre inconnu. L'exemple de ce peintre du Nord est particulièrement intéressant pour notre étude, car il montre comment une accentuation texturale peut faire "remonter" une ou plusieurs dominantes perceptives. Au lieu d'une simple accentuation d'une qualité, en l'occurrence la texture, la variation intentionnelle apparaît comme un changement de perspective qui configure l'objet de sens à partir de la présomption texturale et organise toutes les propriétés autour d'elle.

Lorsqu'il superpose des couches de peinture pour perdre la figure posée initialement, Eugène Leroy compose un objet de sens commandé par la texture, sur une présomption texturale. Pourtant, l'accumulation des épaisseurs de pâte actualise une seconde dominante, l'olfaction, parce que la peinture à l'huile sent si fort qu'elle risque, pour peu qu'on accumule les œuvres en un même endroit, d'incommoder le visiteur.

D'autres dominantes, d'ordre thermique et auditive, apparaissent de même dans une installation polysensorielle de J. Beuys, telle que Plight (1985) du Musée national d'art moderne: deux salles, reliées par un passage bas, qui accueillent un piano, un tableau noir et un thermomètre. Entièrement tapissées d'épais rouleaux de feutre, celles-ci dégagent une chaleur impressionnante et une étrange sonorité.

\section{UNE ACTIVITÉ MÉTADISCURSIVE}

Sur ces exemples intrigants, on voit s'ébaucher une dimension métadiscursive de l'art, une activité réflexive et commentative portant sur sa propre énonciation. En réorganisant l'objet de sens, la peinture s'affirme comme peintureet la sculpture, comme sculpture. Quelle serait la motivation d'une telle activité métalinguistique? I. Rock ${ }^{30}$ émet cette hypothèse invérifiable que l'accentuation de la texture, qu'il observe chez Van Gogh, procède d'un effort de l'œuvre visuelle pour se distinguer du trompe-l'œil, qui n'est pas de l'art. Nous ne nous satisferions pas de la simple ressemblance et exigerions de l'art qu'il se dise tel.
En peinture comme en sculpture, nous pouvons donner quelques exemples d'ajouts à la ressemblance: tel sculpteur a ajouté des côtes au thorax de son modèle ou exagéré l'amplitude d'un mouvement; tel artiste du "pop art», Roy Lichtenstein, rappelle l'origine graphique de son image par un usage hyperbolique du pixel graphique. P. Virilio, par exemple, rapporte les propos de Rodin, qui oppose la représentation du mouvement donnée par la sculpture et le mouvement restitué par la photographie instantanée. L'artiste donne même raison à la sculpture qui, par ses moyens propres, ceux du volume, et en «exagérant» ce dernier, parvient à rendre compte du déroulement du geste dans le temps ${ }^{31}$.

Cette activité métadiscursive apparaît de même dans le graphisme numérique, qui ne cesse de rappeler son modèle génératif en accentuant des effets de texture fondés sur le carré. C'est à ce constat qu'aboutissait en tout cas une étude ${ }^{32}$ du modèle plastique de l'artiste-informaticien John Maeda, qui met en évidence le pixel comme unité programmatique permettant d'élaborer des images publicitaires représentant une Joconde ou un tournesol. En pareils cas, l'hyperbole texturale ne saurait être imputée à une difficulté technique, dans la mesure où le numérique pourrait tout aussi bien dissimuler son origine en estompant la discontinuité du modèle récursif sous une continuité graphique.

\section{DU VISUEL AU TACTILE}

Ces divers exemples, tirés de l'histoire de l'art du $\mathrm{XX}^{\mathrm{e}}$ siècle, font en tout cas de la texture une qualité visuelle qui s'entremêle à la couleur, à la lumière et à l'éidétique. La texture est alors une propriété haptique au sens de Deleuze, relative à la vue qui « découvre (en elle-même) une fonction du toucher qui lui est propre et n'appartient qu'à elle "33. Partagée entre le visuel et le tactile, la texture est néanmoins une propriété accessible au toucher. C'est alors sur elle que se fonde l'installation.

L'éclatement des concordances synesthésiques et l'accentuation différenciée des qualités ancillaires sont 
des constantes de l'installation. Ils renvoient à la définition même de l'installation qui est tantôt une composition, tantôt une configuration au sens de Bordron ${ }^{34}$, selon que ses parties diffèrent en genre ou par l'espèce. Une installation peut associer des peintures et des volumes, y mêler le son ou la photographie, par exemple. Les parties de l'installation peuvent signifier isolément, mais leur sens s'instruit nécessairement de façon globale: elles entretiennent donc une relation de dépendance libre, c'est leur deuxième caractéristique.

Cette hétérogénéité définitoire s'accomplit surtout dans un souci constant de rupture avec les conventions: si l'installation est un genre, c'est un genre fuyant qui défie les catégorisations et les styles, poussé à explorer de nouveaux territoires et soucieux de mettre en cause ses propres codes pour manifester cette «inquiétante étrangeté» qu'ont évoquée plusieurs auteurs ${ }^{35}$. L'hétérogénéité se trouve également confortée par un souci de diversification épistémologique, certaines parties revendiquant une fabrication par l'artiste quand d'autres relèvent plutôt de la tradition des «ready-made» ou, si l'on préfère, des objets trouvés. Ces quelques critères suffisent à faire de l'installation un champ voué à la diversification du sensible, où l'on s'affranchit des routines perceptives et de la «forme générale» des objets.

\section{L'EFFET PASSIONNEL}

Une dernière caractéristique de l'installation, sa composante pathémique, plus rarement aperçue, nous permettra d'entrer dans l'intimité de l'expérience perceptive pour observer l'ébranlement du corps propre.

Dans ses descriptions des installations, la critique d'art évoque souvent un ébranlement, un effondrement. Ce sont les termes mêmes de J. Kristeva à propos d'installations de Hans Haacke et de Bob Wilson visitées à la Biennale de Venise, qu'elle rapporte plus loin à des «maladies de l'âme»:

Lorsque vous êtes devant ces débris, ces objets disséminés, vous ne savez plus qui vous êtes. Vous êtes au bord d'un vertige, d'un trou noir, d'un morcellement de la vie psychique que certains appelleront psychose ou autisme. N'est-ce pas là le redoutable privilège de l'art contemporain que de nous accompagner dans ces nouvelles maladies de l'âme? 36

Or, un lien de causalité pourrait, selon nous, être fait entre cet effet passionnel, ces souffrances du corpspropre si difficiles à appréhender et l'éclatement du sensible. Une telle hypothèse peut être argumentée au moyen d'exemples qui révèlent:

- une mise en cause de la dominante visuelle, à son tour secondarisée;

- la promotion d'une dominante texturale appelant, en certains cas, une seconde modalité ancillaire;

- une modification du rapport au corps du sujet;

- une perturbation du corps propre occasionnant des effets de sens passionnels.

\section{LE PAVILLON ROUGE}

Pourtant présentée dans le cadre de la prestigieuse Biennale de Venise, l'installation que nous prendrons en exemple y est passée largement inaperçue au point que Kristeva, qui officiait pourtant en tant que membre du jury lors de cette Biennale de 1993, ne la mentionne pas à l'appui d'une argumentation dont elle assurait pourtant la meilleure validation ${ }^{37}$. Due à Illja Kabakov et intitulée Le Pavillon rouge, l'installation était présentée dans un pavillon national abandonné aux gravats et aux herbes folles, le pavillon de la CEI, appellation toute fraîche d'une Union soviétique juste défaite.

Cette installation sépare spatialement les modalités visible et tactile: le visiteur traverse le pavillon tout d'abord, dans une obscurité presque parfaite, avant d'accéder au jardin situé à l'arrière du bâtiment, où il découvre, dans la lumière éblouissante de l'été vénitien et sur fond de lagune, un petit kiosque rouge diffusant l'ambiance sonore d'un défilé populaire.

La dichotomie visible/tactile détermine un rapport différencié au corps propre: s'offrant essentiellement à la vue et à l'ouie, sens exotaxiques, l'univers lumineux s'observe dans la distance et de façon globale, tandis que l'univers sombre, à prévalence tactile, est accessible par le contact de la main ou du corps entier 
qui déambule entre cloisons et tas de gravats. Une perception distanciée pour l'univers visible; une proximo-réception pour l'univers tactile; une visée globale et synthétique contre une visée locale et analytique qui perçoit le monde sous forme de fragments.

Une telle séparation spatiale sous-tend en outre un contraste axiologique fondé sur l'opposition clair/ sombre et la rencontre de deux parcours modaux. Le premier trajet s'effectue dans l'obscurité où la profondeur est centrifuge, restreinte, limitée au champ personnel immédiat du visiteur, si ce n'est à son enveloppe corporelle. Quoique rétensive, une telle perception ne se conçoit cependant pas hors d'une protension ${ }^{38}$, dans la mesure où le visiteur, dépourvu des commodités qu'offrirait un champ périphérique, doit "promener» pour ainsi dire son champ perceptif dans la pénombre, saisir des "prises» sur les parois verticales et tâcher d'éviter les gravats pour progresser jusqu'à la sortie.

Si l'on perçoit aisément la dimension pathémique de ce parcours narratif et modal, le visiteur l'hypothétique visiteur -, qui vient d'expérimenter le vaste registre émotionnel des espaces proches, aura sans doute vérifié l'incidence sémantique de ce trajet sur le suivant. De la même façon que le si conservait nécessairement, pour Greimas, la mémoire d'un non antérieur, la perception de l'univers du jardin est en effet sémantisée, "euphorisée» par la périlleuse progression dans le noir qui précède.

\section{L'OBSCURITÉ ET L'ENVELOPPE}

Le Pavillon rouge de Kabakov est à maints égards exemplaire: parce qu'il procède à la séparation des modalités de la façon la plus radicale sans doute, l'obscurité qui sépare visible et tactile défait les synesthésies, instaure l'affect de l'installation et impose le caractère aventureux, hypothétique d'une quête. Lieu commun de l'installation, cette obscurité - le plus souvent une pénombre - s'observe notamment chez Louise Bourgeois (qui place même ses sculptures «traditionnelles», de marbre ou de bois, dans la pénombre). L'efficacité pathémique de l'obscurité se double, plus largement, d'une fonction épistémologique, puisque celle-ci produit un effet de rupture énonciative et fait office de contour d'énoncé. Dépourvue des procédures conventionnelles que sont le cadre pour la peintre et le socle pour la sculpture, l'installation réclame un contour d'énoncé ad hoc qui circonscrira la scène narrative. Tenant lieu de contour, l'obscurité assure ainsi l'insertion d'un alors-ailleurs dans l'isotopie quotidienne et produit ce basculement spatial et temporel bien connu au théâtre: le noir.

Lobscurité assure donc la rupture énonciative qui manque à l'installation: c'est sa fonction la plus élémentaire. Très souvent, elle vient en appui d'un autre dispositif épistémologique, l'enveloppe, comme nous l'avons par ailleurs observé dans les cellules et tanières de L. Bourgeois ${ }^{39}$. Déjà utilisée dans le Merzbau de K. Schwitters, l'enveloppe assure aussi la rupture énonciative d'habitacles, tels Le Jardin d'hiver de J. Dubuffet ou les structures de plâtre d'Absalon.

\section{CONCLUSION}

Lorsque la texture est dégagée de l'équivoque qui tantôt la conçoit comme représentation et tantôt comme ostension, elle s'impose comme un possible fil conducteur de l'art du XXe siècle, de l'art moderne à l'art actuel. En entraînant d'autres dominantes, olfactives, thermiques ou auditives, l'accentuation de la texture permet d'abolir les routines perceptives et, associant les sens autrement, élabore sans cesse d'autres objets signifiants. Passant du visuel au tactile et dominant la peinture comme l'installation, la texture diversifie le sensible en lui ajoutant une épaisseur, une intensité supplémentaire. Sur ce constat, notre plaidoyer en faveur de cette dimension ancillaire rejoint le magnifique hommage rendu par P. Ricœur à l'art de son temps:

[...] c'est lorsque la peinture a cessé, au XXe siècle, d'être figurative que l'on a pu prendre enfin la pleine mesure de [la] mimésis, qui a justement pour fonction, non pas de nous aider à reconnaître des objets, mais à découvrir des dimensions de l'expérience qui n'existaient pas avant l'oeurre. 40 


\section{NOTES}

1. M. Serres, Les Cinq Sens, Paris, Hachette, (1985) 1998, p. 25.

2. E. Cassirer, La Philosophie des formes symboliques, t. 3, Paris, Éd. de Minuit, 1972, p. 89

3. J. Ninio, L'Empreinte des sens. Perception, mémoire, langage, Paris, Odile Jacob, (1989) 1996, p. 61.

4. F. Saint-Martin, Sémiologie du langage visuel, Sillery, Presses de l'Université du Québec, 1987, p. 68.

5. Groupe $\mu$, Traité $d u$ signe visuel. Pour une rhétorique de l'image, Paris, Le Seuil, 1992, p. 70.

6. Pour le Groupe $\mu$, la synesthésie suppose une équivalence entre le tactile et le visuel (voir à ce sujet la note de leur définition, p. 425).

Nous la décririons plus simplement comme une concordance intersensorielle.

7. Ibid.

8. F. Saint-Martin, op.cit., p. 65.

9. Pour les deux conceptions de la couleur, nous renvoyons par exemple à J. Itten, Art de la couleur. Approche subjective et description objective de l'art, Paris, Dessain et Tolra, (1961) 1999, et à la synthèse faite par M. Brusatin, Histoire des couleurs, Paris, Flammarion, (1985) 1996.

10. J. Fontanille, Sémiotique du visible. Des mondes de lumière, Paris, P.U.F., 1995.

11. E. H. Gombrich, L'Art et l'Illusion, Paris, Gallimard, (1971) 1996, p. 280.

12. Groupe $\mu$, op. cit., p. 70-71.

13. J. Maeda, Maeda at Media. Journal d'un explorateur du numérique, Paris, Thames \& Hudson, 2000, p. 250.

14. A. Lhote, Traités du paysage et de la figure, Paris, Grasset, 1978, p. 109.

15. Pour affiner la typologie, on se reportera à M. Baxandall, Ombres et Lumières, Paris, Gallimard, 1993.

16. E. H. Gombrich, Ombres portées, leur représentation dans l'art occidental, Paris, Gallimard, 1995, p. 29.

17. E. H. Gombrich, L'Art et l'Illusion, (1971) 1996, p. 38

18. M. Guillot, Variété des couleurs obtenues en peinture avec un seul pigment. Problèmes de la couleur, p. 169, cité par F. Saint-Martin, op. cit., p. 66. Nous avons souligné.

19. F. Saint-Martin, op. cit., p. 67. L'auteur reprend G. Kanisza.

20. J. Ninio, op.cit., p. 65.

21. F. Saint-Martin, op.cit., p. 23.

22. P. Monnier et L. Saksis, L'CEil, les yeux, cette reproduction, Rome, Villa Médicis, 1993, non paginé.

23. Avec de nombreux auteurs, H. Alekan a montré comment reproduire la fiction de la propagation de la lumière et produire des ombres variables en directivité, en surface et en intensité. Voir à ce sujet H. Alekan, Des lumières et des ombres, Paris, Éd. du collectionneur, 2001, p. 31.

24. J. Ninio, op. cit., p. 61 et suiv.

25. À l'entrée «frottage», le Dictionnaire abrégé du surréalisme précise: «Frottage: procédé découvert par Max Ernst le 10 août 1925. Le procédé de frottage ne reposant que sur l'intensification de l'irritabilité des facultés de l'esprit par des moyens techniques appropriés, excluant toute conduction mentale consciente, réduisant à l'extrême la part active de celui qu'on appelait jusqu'alors "l'auteur", ce procédé s'est révélé le véritable équivalent de l'écriture automatique». B. Éluard, Dictionnaire abrégé du surréalisme, Paris, José Corti, 1995, p. 12. 26. Le Dictionnaire abrégé du surréalisme donne cette définition du collage: «Si ce sont les plumes qui font le plumage, ce n'est pas la colle qui fait le collage» (Max Ernst). «Il est quelque chose comme l'alchimie de l'image visuelle. Le miracle de la transfiguration totale des êtres et objets avec ou sans modification de leur aspect physique ou anatomique" (op. cit., p. 7).

27. G. Deleuze, Différence et Répétition, Paris, P.U.F., (1968) 1989, p. 4. 28. Nous reportons le lecteur à notre texte «Eric Sanchez, les images et les fantômes ", Art présence, n ${ }^{\circ} 43$, juillet-août-septembre 2002, p. 20-23.

29. Nous reportons également à "Plasticité et signification, le cas d'Eugène Leroy", Protée, vol. 27, nº 2 ("La Réception»), p. 125-132. 30. I. Rock, La Perception, Paris, De Boeck, 2001.

31. «Si l'ensemble est faux dans sa simultanéité, concède Rodin, il est vrai quand les parties sont observées successivement, et c'est cette vérité seule qui importe puisque c'est elle que nous voyons et qui nous frappe». Voir à ce sujet P. Virilio, La Machine de vision, Paris, Galilée, 1988, p. 15.

32. A. Beyaert, «L'esthétique du pixel. L'accentuation de la texture dans l'œuvre graphique de John Maeda", Communication et langages (à paraître).

33. G. Deleuze, Logique de la sensation, Paris, Éd. de la différence, 1984, p. 99.

34. Les deux types d'assemblage sont décrits dans J.-F. Bordron, «Les objets en parties. Esquisse d'ontologie matérielle», dans J.-C. Coquet et J. Petitot (sous la dir. de), Langages, $\mathrm{n}^{\circ} 3$ ("L'Objet sens et réalité»), septembre 1991, p. 58.

35. Je me réfère notamment à $M$. Regimbald, «L'installation, cette inquiétante étrangère", Espace (sculpture), no 17, automne 1991, p. 6-11. 36. J. Kristeva, Sens et non-sens de la révolte. Pouvoirs et limites de la psychanalyse I, Paris, Fayard, 1996, p. 52.

37. Dans le catalogue de la Biennale, l'œuvre est laconiquement mentionnée par son titre et un croquis, la photographie d'illustration représentant une installation antérieure de Kabakov, Les Toilettes. Voir à ce sujet XLV esposizione internazionale d'arte, Venise, Éd. Marsili, 1993, p. 104-105.

38. "Quand la main est en contact avec l'objet-cible, elle ne dispose pas, comme dans le système oculaire d'un "champ périphérique" ayant une valeur d'appel et pouvant fournir des points d'ancrage. Le sujet doit donc effectuer intentionnellement des mouvements d'exploration dans l'espace de travail pour chercher (s'ils existent) des repères extérieurs ", dans Y. Hatwell et alii, Toucher pour connaitre. Psychologie cognitive de la perception tactile manuelle, Paris, P.U.F., 2000, p.2. 39. A. Beyaert, «Retiens la nuit. L'obscurité dans l'installation contemporaine", Voir barré, nos 24-25 ("Figures de la nuit»), décembre 2002, p. 40-51.

40. P. Ricœur, La Critique et la Conviction: entretien avec François Azouvi et Marc de Launay, Paris, Calmann-Lévy, 1995, p. 260. 